Подригало М.А., Тарасов Ю.В.

Харьковский национальный автомобильно-дорожный университет

\title{
ПРИМЕНЕНИЕ МЕТОДА ПАРЦИАЛЬНЫХ УСКОРЕНИЙ ДЛЯ ОЦЕНКИ ФУНКЦИОНАЛЬНОЙ СТАБИЛЬНОСТИ ДИНАМИЧЕСКИХ СВОЙСТВ АВТОМОБИЛЯ
}

\footnotetext{
В данной статье представлена методическая база для оценки функциональной стабильности динамических свойств автомобиля в процессе эксплуатации путем применения метода парциальных ускорений. Следует отметить, что динамика изменения величины парциальных ускорений может характеризовать параметрическую стабильность, а скорость падения величины ускорения функциональную стабильность автомобиля (его динамических свойств). Предложен критерий для оценки функциональной стабильности динамических свойств автомобиля на основе величины максимального ускорения автомобиля по пробегу в эксплуатации.

Ключевые слова: автомобиль, параметрическая стабильность, парциальное ускорение, функциональная стабильность, сопротивление движению, эксплуатация автомобиля, дополнительные потери энергии.
}

Постановка проблемы. Ухудшение динамических свойств, вызванное нестабильностью параметров автомобиля, является причиной дорожно-транспортных происшествий, в том числе и с человеческими жертвами [1].

Для обеспечения безопасности движения необходимо либо проводить контрольные динамические испытания автомобилей на функциональную стабильность, либо осуществлять оценку динамики изменения контрольных параметров в процессе эксплуатации с помощью бортового измерительного комплекса. Существующие показатели динамических свойств автотранспортных средств не полностью учитывают факторы, которые влияют на современные автомобили. Данные недостатки приводят к ошибкам не только при разработке нормативов, но и при их выборе для конкретных условий, поскольку неучтенные показатели предопределяют ошибки в оценке технического уровня автомобилей. Применение не уточненных показателей динамических свойств автотранспортных средств для управления техническим состоянием автомобилей влечет за собой материальный ущерб в связи с необоснованным повышением затрат при несвоевременном техническом обслуживании (ТО) и ремонте.

В настоящей статье, с использованием метода парциальных ускорений, предложен алгоритм решения указанной проблемы.

Анализ последних достижений и публикаций. Метод парциальных ускорений представляет собой решение прямой (1-й) задачи механики, т.е. задачи определения действующих сил по заданному движению тела [2].

Метод парциальных ускорений построен на переходе от векторной суммы в пространстве сил к векторной сумме в пространстве ускорений [2]. Векторная сумма ускорений может рассматриваться как сумма членов ряда, каждый из которых представляет собой некоторую зависимость от скорости автомобиля $[3,4,5]$. В основе метода лежит принцип суперпозиции в механике. Если по характеру приведения векторов принцип Д'Аламбера называют принципом кинетостатики, то предлагаемый метод парциальных ускорений следует считать принципом кинетодинамики. Если принцип Д’Аламбера удобен при проведении теоретических исследований, то метод парциальных ускорений удобен при проведении экспериментальных исследований и дальнейшей обработки их результатов и обобщений [3].

В работе [3] указанный ряд назван уравнением парциальных ускорений автомобиля и для случая выбега представлен в следующем виде:

$$
\dot{V}_{a}=-g \psi-\frac{M_{\text {ТРСБ }}^{C}}{m_{a} r_{\partial}}+\frac{M_{\text {ТРКИН }}^{C}}{m_{a} r_{\partial}}+\frac{M_{\text {ТРДИН }}^{C}}{m_{a} r_{\partial}}-\frac{A_{W} \rho F}{2 m_{a}} V_{a}^{2-n},
$$

где $\dot{V}_{a}$ - линейное ускорение автомобиля при выбеге $\left(\dot{V}_{a}<0\right)$;

$V_{a}$-линейная скорость автомобиля; 
$\psi$ - коэффициент суммарного дорожного сопротивления;

$i$ - продольный уклон дороги;

$$
\psi=f \pm i
$$

$M_{T P . C T}^{C}-$ статический момент сопротивления в трансмиссии, обусловленный силами сухого трения;

$M_{\text {TP.Кин }}^{C}$ момент сопротивления в трансмиссии, обусловленный силами вязкого трения (кинематический);

$$
M_{\text {TP.Кин }}^{C}=-K_{1} V_{a}
$$

$\kappa_{1}$ - коэффициент пропорциональности между скоростью $V_{a}$ автомобиля и моментом

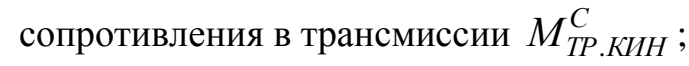

$M_{T P . д и H^{-}}^{C}$ момент сопротивления в трансмиссии, обусловленный разгоном инерционных масс и зависящий от ускорения автомобиля $V_{a}$;

$$
M_{T P . д и н ~}^{C}=-\frac{J_{\Pi P}^{T P}}{r_{l}} \dot{V}_{a}
$$

$m_{a}$ - масса автомобиля;

$A_{W}-$ коэффициент, численно равный лобовому аэродинамическому сопротивлению автомобиля при $V_{a}=1 \mathrm{м} / \mathrm{c}$; размерность указанного коэффициента $(\mathrm{м} / \mathrm{c})^{\mathrm{n}}[3]$

$n$ - показатель степени, постоянный для данной формы кузова автомобиля [3]

$\rho$ - плотность воздуха;

$F$ - площадь лобового миделевого сечения автомобиля;

$r_{\partial}$ - динамический радиус колес автомобиля;

$J_{П P}^{T P}$ - приведенный к ведущим колесам момент инерции трансмиссии (при отключенном двигателе).

После подстановки выражений (2) - (4) в уравнение (1) получим

$$
\dot{V}_{a}=-g(f \pm i)-\frac{M_{T P . C T}^{C}}{m_{a} r_{\partial}}-\frac{\kappa_{1}}{m_{a} r_{\partial}} V_{a}-\frac{J_{\Pi P}^{T P}}{m_{a} r_{\partial}} \dot{V}_{a}-\frac{A_{W} \rho F}{2 m_{a}} V_{a}^{2}
$$

Разрешив уравнение (5) относительно $\dot{V}_{a}$, находим [2]

$$
\dot{V}_{a}=-\frac{g}{\delta_{\sigma p}^{\prime}}(f \pm i)-\frac{M_{T P . C T}^{C}}{\delta_{\sigma p}^{\prime} m_{a} r}-\frac{\kappa_{1}}{m_{a} r_{\partial}} V_{a}-\frac{J_{\Pi P}^{T P}}{\delta_{\theta p}^{\prime} m_{a} r_{\partial}} \dot{V}_{a}-\frac{A_{W} \rho F}{2 \delta_{\theta p}^{\prime} m_{a}} V_{a}^{2-n}=-a_{1}-a_{2} V_{a}-a_{3} V_{a}^{2-n},(6)
$$

где $\delta_{\epsilon p}^{\prime}$ - коэффициент учета вращающихся масс трансмиссии при отключенном двигателе;

$$
\delta_{\text {ep }}^{\prime}=1+\frac{J_{\Pi P}^{T P}}{m_{a} r_{\partial}},
$$

$a_{1}, a_{2}, a_{3}-$ коэффициенты,

$$
\begin{gathered}
a_{1}=-\frac{g}{\delta_{\theta p}^{\prime}}(f \pm i)-\frac{M_{T P . C T}^{C}}{\delta_{\theta p}^{\prime} m_{a} r_{\partial}} ; \\
a_{2}=\frac{\kappa_{1}}{m_{a} r_{\partial}} ;
\end{gathered}
$$




$$
a_{3}=\frac{A_{W} \rho F}{2 \delta^{\prime} m_{a}} \text {. }
$$

В работе [3] учитывалась также зависимость коэффициента сопротивления качению от скорости [6], имеющая вид

$$
f=f_{0}\left(1+A V_{a}^{2}\right)
$$

Однако, мы в наших дальнейших исследованиях примем $f=f_{0}=$ const. Все члены ряда ускорений (6) являются парциальными ускорениями, характеризующими конкретные суммарные силы сопротивления движению автомобиля. Сумма членов ряда равна ускорению автомобиля при выбеге, которое при рассмотрении движения в тяговом режиме будет являться суммарным парциальным ускорением сил сопротивления движению.

В работе [3] на основе уравнения (6) парциальных ускорений был предложен расчетно экспериментальный метод определения параметров сопротивления движению, кинематических, динамических и мощностных показателей автомобиля.

В тяговом режиме [3] уравнение динамики поступательного движения автомобиля имеет вид

$$
m_{a} \dot{V}_{a}=\frac{N_{e}}{V_{a}}-\frac{M_{T P . C T}^{C}}{r_{\partial}}-\frac{\kappa_{1}}{r_{\partial}} V_{a}-J_{\Pi P} \frac{\dot{V}_{a}}{r_{\partial}}-m_{a} g\left(f_{0} \pm i\right)-\frac{A_{W} \rho F}{2} V_{a}^{2-n},
$$

где $J_{П Р}-$ приведенный к ведущим колесам момент инерции вращающихся масс трансмиссии и двигателя;

$N_{e}$ - эффективная мощность двигателя.

Из уравнения (12) определим ускорение автомобиля

$$
\dot{V}_{a}=\frac{N_{e}}{m_{a} V_{a} b_{1}}-\frac{b_{2}}{b_{1}}-\frac{b_{3}}{b_{1}} V_{a}-\frac{b_{4}}{b_{1}} V_{a}^{2-n},
$$

где $b_{1} ; b_{2} ; b_{3} ; b_{4}$ - коэффициенты, определяемые в процессе движения автомобиля,

$$
\begin{gathered}
b_{1}=\delta_{\theta p} ; \\
b_{2}=g(f \pm i)+\frac{M_{T P . C T}^{C}}{m_{a} r_{\partial}} ; \\
b_{3}=\frac{\kappa_{1}}{m_{a} r_{\partial}} ; \\
b_{4}=\frac{A_{W}}{2 m_{a}} \delta F .
\end{gathered}
$$

Каждый из членов уравнения в правой части (13) является парциальным ускорением. Кроме парциальных ускорений, характеризующих силы сопротивления движению, в уравнении (13) присутствует парциальное ускорение от сил движущих

$$
\dot{V}_{\partial B}^{\text {Iapu }}=\frac{N_{e}}{m_{a} V_{a} b_{1}} .
$$

Таким образом, уравнение (13) можно представить в виде суммы членов ряда парциальных ускорений [3]

$$
\dot{V}_{a}=\dot{V}_{\partial в}^{\text {Пари }}-\dot{V}_{c m}^{\text {Пари }}-\dot{V}_{\text {кин }}^{\text {Пари }}-\dot{V}_{w}^{\text {Пари }} ;
$$

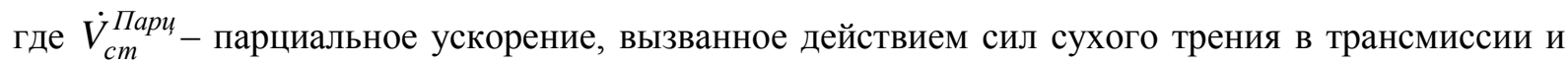
силами суммарного дорожного сопротивления;

$$
\dot{V}_{c m}^{\text {Пapu }}=\frac{g}{\delta_{\theta p}}(f \pm i)+\frac{M_{T P . C T}^{C}}{\delta_{\theta p} m_{a} r \partial} ;
$$


$\dot{V}_{\text {кин }}^{\text {Пари }}$ - парциальное ускорение, вызванное действием сил вязкого трения в трансмиссии;

$$
\dot{V}_{\kappa и н}^{\Pi а р и}=\frac{\kappa_{1}}{\delta_{\text {вp }} m_{a} r_{\partial}} V_{a}
$$

$\dot{V}_{w}^{\text {Пари }}$ - парциальное ускорение, обусловленное силами аэродинамического сопротивления,

$$
\dot{V}_{w}^{\Pi а р u}=\frac{A_{W}}{2 m_{a} \delta_{\theta p}} \delta F V_{a}^{2-n} .
$$

Каждое их парциальных ускорений создается либо движущими силами, либо силами сопротивления движению. Указанные силы ввиду нестабильности параметров автомобиля и изменения величин внешних параметров.

Поэтому парциальные ускорения могут являться контрольными параметрами при оценке функциональной стабильности динамических свойств автомобиля.

Целью исследования является разработка методической базы для оценки функциональной стабильности динамических свойств автомобиля в процессе эксплуатации путем использования метода парциальных ускорений.

Для достижения поставленной цели необходимо решить следующую задачу:

- разработать научно- методологическую базу для оценки параметрической и функциональной стабильности динамических свойств автомобиля.

Результаты исследований. Анализируя выражения (18), (20), (21) и (22) для определения парциальных ускорений, можно сделать выводы о том, что каждое из них является функцией, характеризующей техническое состояние отдельных составных частей автомобиля. Парциальное ускорение от сил движущих $\dot{V}_{\partial s}^{\text {Пари }}$ характеризует техническое состояние и стабильность параметров двигателя. Парциальное ускорение $\dot{V}_{c m}^{\text {Пари }}$, зависящее от скорости $V_{a}$ автомобиля в нулевой степени, характеризует техническое состояние трансмиссии (изменение ее параметров сухого трения), а также - изменения ходовой части (геометрии, смещения мостов, параметров подвески). В работах [7, 8] предложены методы, позволяющие определять массу автомобиля и продольный уклон дороги в процессе движения с использованием бортовой измерительной системы. Это позволяет учесть массу автомобиля в уравнениях (18), (20), (21) и (22) и продольный уклон дороги в уравнении (20).

Парциальное ускорение $\dot{V}_{\kappa и н ~}^{\text {Пари }}$ (выражение $\left.(21)\right)$ характеризует вязкость и уровень масла в трансмиссии автомобиля. Парциальное ускорение $\dot{V}_{w}^{\text {Пари }}$ является наиболее стабильным, изменяющимся в зависимости от загрузки и установки на автомобиле дополнительного оборудования. Изменение массы, как уже отмечалось, можно регистрировать с помощью бортового измерительного комплекса, установленного на автомобиле. При установке внешнего багажника на крыше автомобиля в память бортового компьютера должны вводиться параметры увеличенного аэродинамического сопротивления.

В работе [3] приведен алгоритм расчета параметров $\dot{V}_{\partial s}^{\text {Пари }}, \dot{V}_{\kappa u н}^{\text {Пари }}, \dot{V}_{c m}^{\text {Пари }}, \dot{V}_{w}^{\text {Пари }}$ в процессе движения автомобиля. При накоплении значений указанных величин, получаемых при периодических тестовых замерах, возможна оценка динамики парциальных ускорений и функциональной и параметрической стабильности автомобиля.

Для $i$-го парциального ускорения показателем стабильности будет являться производная указанного ускорения по наработке автомобиля $\frac{\partial \dot{V}_{i}^{\text {Пари }}}{\partial L}$. Критерием сохранения стабильности является максимально допустимая скорость изменения указанного і-го парциального ускорения (его величины). Очевидно, что с увеличением наработки парциальное ускорение от сил движущих $\dot{V}_{\partial в}^{\text {Пари }}$ уменьшается, а остальные ускорения увеличиваются по абсолютной величине (кроме $\dot{V}_{w}^{\text {Пари }}$ ). Таким образом

$$
\begin{aligned}
& \frac{\partial \dot{V}_{i}^{\Pi a p u}}{\partial L}<0 \\
& \partial \dot{V}_{c m}^{\Pi a p u}>0 ;
\end{aligned}
$$




$$
\begin{aligned}
& \frac{\partial \dot{V}_{\text {Кини }}^{\text {Пари }}}{\partial L}>0 ; \\
& \frac{\partial \dot{V}_{W}^{\text {Пари }}}{\partial L}=0 ;
\end{aligned}
$$

где $L$ - пробег автомобиля.

При

$$
\begin{aligned}
& \dot{V}_{\partial \varepsilon}^{\text {Пapu }}<\left[\dot{V}_{\partial \varepsilon}^{\text {Пapu }}\right]_{\min } ; \\
& \dot{V}_{c m}^{\text {Пари }}>\left[\dot{V}_{c m}^{\text {Парu }}\right]_{\max } \text {; } \\
& \dot{V}_{\text {кин }}^{\text {Пари }}>\left[\dot{V}_{\text {кин }}^{\text {Пари }}\right]_{\max } \text {. }
\end{aligned}
$$

дальнейшая эксплуатация автомобиля без ТО и ремонта недопустима. В неравенствах (27) - (29) представлены следующие величины: $\left[\dot{V}_{\partial \varepsilon}^{\text {Пари }}\right]_{\min }-$ минимально допустимое парциальное ускорение от сил движущих; $\left[\dot{V}_{c m}^{\text {Пари }}\right]_{\max } ;\left[\dot{V}_{\text {кин }}^{\text {Пари }}\right]_{\max }$ - максимально допустимые парциальные ускорения от сил сопротивления. В соответствии с уравнением (19) при увеличении пробега $L$ автомобиля происходит уменьшение ускорения автомобиля. Поэтому $\dot{V}_{a}$ может являться показателем функциональной стабильности динамических свойств и использоваться при проведении контрольных испытаний.

Следует отметить, что динамика изменения величины парциальных ускорений может характеризовать параметрическую стабильность, а скорость падения величины ускорения $\dot{V}_{a}-$ функциональную стабильность автомобиля (его динамических свойств).

Выводы. Изменения величины парциальных ускорений в процессе эксплуатации могут являться показателями параметрической стабильности динамических свойств автомобиля. Скорость изменения величин парциальных ускорений по пробегу машины может нормироваться при оценке указанной стабильности динамических свойств.

Скорость падения величины максимального ускорения автомобиля по пробегу может являться показателем функциональной стабильности его динамических свойств и также нормироваться.

1.Динамика автомобиля / М. А. Подригало, В. П. Волков, А. А. Бобошко, В. А. Павленко, В. Л. Файст, Д. М. Клец, В. В. Редько / Под ред. М. А. Подригало. - Харьков: Изд-во ХНАДУ, 2008. 424 с.

2.Тарг С.М. Краткий курс теоретической механики/ С.М. Тарг. - М.:Наука, 1968. - 480 с.

3.Метод парциальных ускорений и его приложения в динамике мобильных машин / [Н.П.Артемов, А.Т.Лебедев, М.А.Подригало, А.С.Полянский, Д.М.Клец, А.И.Коробко, В.В.Задорожняя ]; под ред. М.А. Подригало - Х.:Міськдрук, 2012. $-220 \mathrm{c}$.

4. Коробко А. И. Метод определения энергетических и динамических показателей автомобиля с помощью датчиков линейных ускорений / Подригало М. А., Клец Д. М., Мостовая А. Н., Коробко А. И. // Вісник Східноукраїнського національного університету імені Володимира Даля. Науковий журнал. - 2010. - № 7 (149). - С. 40-44.

5. Коробко А. І. Застосування методу паралельних спостережень при випробуваннях автомобілів / Михайло Подригало, Андрій Коробко // Актуальні задачі сучасних технологій : міжнародна науково-технічна конференція молодих учених та студентів, 21-22 грудня 2010 р. : збірник тез доповідей. - Тернопіль, 2010. - С. 60.

6.Бортницкий П.И. Тягово - скоростные качества автомобилей. Справочник / П.И. Бортницкий, В.И. Задорожный. К.: Вища школа, 1978. - 176 с.

7.Абрамов Д.В. Концепція покращення функціональної стабільності динамічних та енергоперетворюючих властивостей автомобілів: диссертиція на здобуття накового ступеня доктора технічних наук: 05.22 .02 / Абрамов Дмитро Володимирович. - Х., 2018. -480 с.

8. Коробко А. І. Удосконалення методу визначення компонентів сил опору руху автомобіля / М. А. Подригало, А. І. Коробко // Новітні технології - для захисту повітряного простору : сьома наукова конференція Харківського університету повітряних сил імені Івана Кожедуба, 13-14 квітня 2011 р. : тези доповідей. - Харків, 2011. - С. 188.

\section{REFERENCES}

1.Dinamika avtomobilya / M. A. Podrigalo, V. P. Volkov, A. A. Boboshko, V. A. Pavlenko, V. L. Fajst, D. M. Klec, V. V. Red'ko / Pod red. M. A. Podrigalo. - Har'kov: Izd-vo HNADU, 2008. 424 s.

2.Targ S.M. Kratkij kurs teoreticheskoj mekhaniki/ S.M. Targ. - M.:Nauka, 1968. $-480 \mathrm{~s}$.

3.Metod parcial'nyh uskorenij i ego prilozheniya $\mathrm{v}$ dinamike mobil'nyh mashin / [N.P.Artemov, A.T.Lebedev, M.A.Podrigalo, A.S.Polyanskij, D.M.Klec, A.I.Korobko, V.V.Zadorozhnyaya ]; pod red. M.A. Podrigalo - H.:Mis'kdruk, 2012. $220 \mathrm{~s}$. 
4. Korobko A. I. Metod opredeleniya energeticheskih i dinamicheskih pokazatelej avtomobilya s pomoshch'yu davachov linejnyh uskorenij / Podrigalo M. A., Klec D. M., Mostovaya A. N., Korobko A. I. // Visnik Skhidnoukraïns'kogo nacional'nogo universitetu imeni Volodimira Dalya. Naukovij zhurnal. - 2010. - № 7 (149). - S. 40-44.

5. Korobko A. I. Zastosuvannya metodu paralel'nih sposterezhen' pri viprobuvannyah avtomobiliv / Mihajlo Podrigalo, Andrij Korobko // Aktual'ni zadachi suchasnih tekhnologij : mizhnarodna naukovo-tekhnichna konferenciya molodih uchenih ta studentiv, 21-22 grudnya 2010 r. : zbirnik tez dopovidej. - Ternopil', 2010. - S. 60.

6.Bortnickij P.I. Tyagovo - skorostnye kachestva avtomobilej. Spravochnik / P.I. Bortnickij, V.I. Zadorozhnyj. - K.: Vishcha shkola, 1978. - $176 \mathrm{~s}$.

7.Abramov D.V. Koncepciya pokrashchennya funkcional'noï stabil'nosti dinamichnih ta energoperetvoryuyuchih vlastivostej avtomobiliv: disserticiya na zdobuttya nakovogo stupenya doktora tekhnichnih nauk: 05.22.02 / Abramov Dmitro Volodimirovich. H., 2018. $-480 \mathrm{~s}$.

8. Korobko A. I. Udoskonalennya metodu viznachennya komponentiv sil oporu ruhu avtomobilya / M. A. Podrigalo, A. I. Korobko // Novitni tekhnologiï - dlya zahistu povitryanogo prostoru : s'oma naukova konferenciya Harkivs'kogo universitetu povitryanih sil imeni Ivana Kozheduba, 13-14 kvitnya 2011 r. : tezi dopovidej. - Harkiv, 2011. - S. 188.

Подригало М.А., Тарасов Ю.В. Застосування методу парціальних прискорень для оцінки функціональної стабільності динамічних властивостей автомобіля

У даній статті представлена методична база для оцінки функціональної стабільності динамічних властивостей автомобіля в процесі експлуатації шляхом застосування методу парціальних прискорень. Слід зазначити, що динаміка зміни величини парціальних прискорень може характеризувати параметричну стабільність, а швидкість падіння величини прискорення функціональну стабільність автомобіля (його динамічних властивостей). Запропоновано критерій для оцінки функціональної стабільності динамічних властивостей автомобіля на основі величини максимального прискорення автомобіля по пробігу в експлуатації.

Ключові слова: автомобіль, параметрична стабільність, парціальние прискорення, функціональна стабільність, опір руху, експлуатація автомобіля, додаткові втрати енергії.

Podrigalo MA, Tarasov Yu.V. Application of the partial acceleration method to assess the functional stability of the dynamic properties of the car

This article presents a methodological basis for assessing the functional stability of the dynamic properties of a car during operation by applying the method of partial accelerations. It should be noted that the dynamics of changes in the magnitude of partial accelerations can characterize parametric stability, and the rate of fall in the magnitude of acceleration is the functional stability of the vehicle (its dynamic properties). A criterion for assessing the functional stability of the dynamic properties of the car based on the maximum acceleration of the car over the mileage in operation is proposed.

Keywords: automobile, parametric stability, partial acceleration, functional stability, resistance to movement, vehicle operation, additional energy loss.

ПОДРИГАЛО Михайло Абович, доктор технічних наук, професор, завідувач кафедри «Технології машинобудування і ремонту машин» Харківський національний автомобільно-дорожній університет, e-mail: pmikhab@gmail.com

ТАРАСОВ Юрій Володимирович, кандидат технічних наук, доцент кафедри «Технології машинобудування і ремонту машин» Харківський національний автомобільно-дорожній університет, e-mail: yuriy.ledd@gmail.com

ПОДРИГАЛО Михаил Абович, доктор технических наук, профессор, заведующий кафедры «Технологии машиностроения и ремонта машин» Харьковский национальный автомобильнодорожный университет, e-mail: pmikhab@gmail.com

ТАРАСОВ Юрий Владимирович, кандидат технических наук, доцент кафедры «Технологии машиностроения и ремонта машин» Харьковский национальный автомобильно-дорожный университет, e-mail: yuriy.ledd@gmail.com

Mykhaylo PODRYHALO, Doctor of Science in Engineering, Professor, Head of the Department of Engineering Technology and Machine Repair Kharkov National Automobile and Highway University, email: pmikhab@gmail.com

Yurii TARASOV, PhD. in Engineering, Assoc. Professor of machine building technology and machines repair, Kharkiv National Automobile and Highway University, e-mail: yuriy.ledd@ gmail.com 